\title{
Deformation simulation of cells seeded on a collagen-GAG scaffold in a flow perfusion bioreactor using a sequential 3D CFD-elastostatics model
}

\author{
Jungreuthmayer $\mathrm{C}^{1,2,3}$, Jaasma $\mathrm{MJ}^{1,2}$, Al-Munajjed $A A^{1,2}$, Zanghellini $\mathrm{J}^{4}$, \\ Kelly DJ², O'Brien FJ ${ }^{1,2}$ \\ ${ }^{1}$ Department of Anatomy, Royal College of Surgeons in Ireland, Ireland \\ ${ }^{2}$ Trinity Centre for Bioengineering, Dept of Mechanical and Manufacturing Engineering, Trinity College \\ Dublin, Ireland \\ ${ }^{3}$ Siemens Medical Ireland, Leeson Close, Dublin 2, Ireland \\ ${ }^{4}$ Institute of Chemistry, Karl Franzens University Graz, Austria
}

Address Correspondence and Reprints Requests to:

Professor Fergal J. O'Brien, PhD

Department of Anatomy

Royal College of Surgeons in Ireland

123 St. Stephen's Green

Dublin 2

Ireland

Phone: +353-(0)1-402-2149

Fax: +353-(0)1-402-2355

Email: fjobrien@rcsi.ie 


\section{Abstract}

Tissue-engineered bone shows promise in meeting the huge demand for bone grafts caused by up to 4 million bone replacement procedures per year, worldwide. State-of-the-art bone tissue engineering strategies use flow perfusion bioreactors to apply biophysical stimuli to cells seeded on scaffolds and to grow tissue suitable for implantation into the patient's body. The aim of this study was to quantify the deformation of cells seeded on a collagen-GAG scaffold which was perfused by culture medium inside a flow perfusion bioreactor. Using a $\mu \mathrm{CT}$ scan of an unseeded collagen-GAG scaffold, a sequential 3D CFD-deformation model was developed. The wall shear stress and the hydrostatic wall pressure acting on the cells were computed through the use of a CFD simulation and fed into a linear elastostatics model in order to calculate the deformation of the cells. The model used numerically seeded cells of two common morphologies where cells are either attached flatly on the scaffold wall or bridging two struts of the scaffold. Our study showed that the displacement of the cells is primarily determined by the cell morphology. Although cells of both attachment profiles were subjected to the same mechanical load, cells bridging two struts experienced a deformation up to 500 times higher than cells only attached to one strut. As the scaffold's pore size determines both the mechanical load and the type of attachment, the design of an optimal scaffold must take into account the interplay of these two features and requires a design process that optimizes both parameters at the same time. 


\section{Introduction}

Every year, up to 4 million bone replacement procedures are performed worldwide which require the use of a bone graft [1]. However, both of the most common treatments show substantial drawbacks. Autografts, where bone is taken from the patient's own body and then re-implanted, has only limited availability and an additional invasive surgery is necessary which raises the possibility of donor site morbidity. Allograft, where bone is removed from an organ donor, possesses a small risk of disease transmission and again has limited availability. Therefore, the recent focus of bone graft research has switched to bone tissue engineering, where cells (taken from the patient's bone marrow) are seeded onto a biological scaffold. These cells produce bone tissue in vitro [2] using chemical and biological growth factors or by responding to biophysical stimuli applied by a bioreactor.

Scaffolds play a key role in tissue engineering and must meet various demands. The scaffold material has to be biodegradable and the products of degradation should be non-toxic [3]. The scaffold structure has to be highly porous with a high interconnectivity and a surface area [4] which allows nutrient flow throughout the scaffold and the surrounding host tissue. Collagenglycosaminoglycan (GAG) scaffolds developed by Yannas et al. [5] and adapted for bone tissue engineering by O’Brien et al [6] fulfil all these key criteria and show promising results in vitro [7] and in vivo [8].

The only disadvantage of the collagen-GAG scaffold for bone tissue engineering is that it has relatively poor mechanical properties. However, the mechanical properties of the cell-seeded collagen-GAG scaffold can be improved through in vitro matrix production and mineralisation. Biophysical stimuli can be used to increase this matrix production and improve the levels of mineralisation. One way of applying biophysical stimuli is through the use of a flow perfusion bioreactor as shown in Figure 1, where culture medium is pumped through the cell-seeded scaffold $[9,10,11]$ exposing the cells to a shear stress.

Although several biophysical stimuli have been proposed such as deviatoric stress, hydrostatic 
stress, and principal strain [29,30] it has been established that shear stress is the main biophysical stimulus which causes cells to activate matrix production and mineralization $[12,17,31]$. The applied wall shear stress needs to be in a physiologically relevant range. In vivo, bone cells experience estimated shear stresses of $0.8 \mathrm{~Pa}$ to 3.0Pa for the range of routine physical activity [32]. Shear stress values outside the physiologically relevant range might lead to a lack of osteogenic stimulation or a stimulation of osteogenic activity that is sub-optimal. A number of studies have estimated the levels of shear stress required to stimulate osteoblasts to upregulate osteogenic markers and produce extracellular matrix. Smalt et al. [13] reported that wall shear stress values of 3Pa caused osteoblastic cells seeded on a substrate to release the early bone formation marker prostaglandin $\mathrm{E}_{2}$ but that levels of shear stress as low as $30 \mathrm{mPa}$ did not result in a significant increase of prostaglandin $\mathrm{E}_{2}$. A calcium phosphate scaffold (pore size $~ 350 \mu \mathrm{m}$ ) seeded with MC3T3 cells were used by Vance et at. [14] to stimulate the cells to release prostaglandin $E_{2}$ by applying wall shear stress values of up to 1.2Pa using a flow perfusion bioreactor. However, Jaasma and O’Brien [22] reported that much lower wall shear stress values ( $20 \mathrm{mPa})$ are sufficient to stimulate osteoblastic cells seeded on collagen-GAG scaffolds (pore size $\sim 96 \mu \mathrm{m}$ ) to release prostaglandin $\mathrm{E}_{2}$. Besides the fluid flow, the cell morphology and the cell's position within the scaffold also affect the mechanical response [18]. This indicates that the applied wall shear stress alone is an inadequate measure to determine the optimal biophysical stimulus. We hypothesize that the cell deformation is more suitable to characterize the applied biophysical stimulus, because it not only takes into account the acting forces caused by the fluid flow, but also the cell morphology. Computational fluid-dynamics models (CFD) have been successfully used to quantify the shear stresses acting inside microstructures [15,16,34], whereas finite element deformation simulations have been used to quantify the deformation of cells caused by a fluid flow [17]. Little work has been done in combining these two methods to determine the exact fluid conditions inside a scaffold and calculate its mechanical effect on the seeded cells.. The objective of this study was to determine the cell-level biophysical stimuli within collagen-GAG scaffolds subjected to externally applied 
fluid flow using a bioractor by quantifying the mechanical deformation of the cells. We hypothesise that the different cell attachment profiles to the scaffold struts that are observed experimentally [19] will determine the levels of cell deformation within the scaffold. To investigate this hypothesis, a novel 3D CFD-elastostatics model of a cell-seeded collagen-GAG scaffold was developed to (i) quantify the velocity, the shear stress, and the hydrostatic pressure of the fluid inside the scaffold, to (ii) determine the wall shear stress and the hydrostatic wall pressure, that cells seeded on the scaffold are exposed to, and to (iii) analyse the deformation of cells of two common cell attachment profiles. 


\section{Methods}

A computational model was designed to characterize the deformation of osteoblastic cells seeded on a collagen-GAG scaffold [6] exposed to flow perfusion in a bioreactor. The scaffolds were fabricated in our laboratory [20] and had an average pore size of $96 \mu \mathrm{m}$ [6]. 2x10 ${ }^{6}$ cells were seeded on the scaffold (diameter $=\sim 12.0 \mathrm{~mm}$, thickness $=\sim 3.5 \mathrm{~mm}$ ) as described in [11].

The development of the model required a five step procedure: geometry reconstruction, numerical cell seeding, mesh creation, CFD simulation, and elastostatic simulation.

Step 1: A micro-computed tomography ( $\mu \mathrm{CT}$ ) scan of an unseeded collagen-GAG scaffold was used to obtain a numerical model of the scaffold. The $\mu$ CT scan was performed by SCANCO Medical

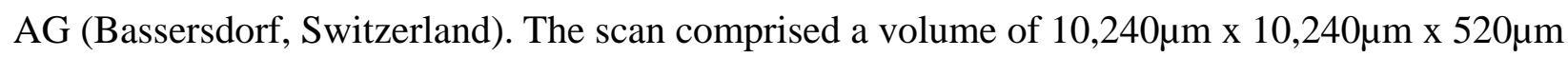
(Figure 2). The pixel size was $5 \mu \mathrm{m} \times 5 \mu \mathrm{m} \times 5 \mu \mathrm{m}$. In order to reduce the computational costs, three randomly chosen sub-volumes with dimensions of $640 \mu \mathrm{m}$ x $640 \mu \mathrm{m}$ x $480 \mu \mathrm{m}$ were analysed. The 3D model of the scaffold geometry was reconstructed from the grey-scale $\mu \mathrm{CT}$ raw data by extracting the chosen sub-volumes, filtering (Gaussian rank filter) and thresholding. Step 2: A computer program was developed to numerically seed cells on the 3D model of the scaffold obtained in Step 1. Previous investigations using confocal microscopy, differentiate two types of cell attachment in the collagen scaffolds used in our laboratory [19]: approximately 25\% of the cells are attached flatly to the scaffold wall and $75 \%$ of the cells are attached bridging two struts of the scaffold [19,35]. The seeding program was able to seed both types of cells on the 3D model. The seeded cells were between 45 and $65 \mu \mathrm{m}$ long. Cells attached to two struts had the form of a sphere representing the cell body and two processes. The average diameter of the sphere was $15 \mu \mathrm{m}$ and the average diameter of the cylinder-shaped processes was 2.2 $\mu \mathrm{m}$ [21] (see Figure 3a). Cells flatly attached to the scaffold wall had the form of a hemisphere representing the cell body (see Figure 3b) and two processes with a semi-circular cross section. In order to obtain the same cell volume for both cell types, the average diameter of the hemispherical-shaped cell body was $19 \mu \mathrm{m}$ and the average diameter of the semi-circular processes was 3.0 $\mu \mathrm{m}$. The seeding program used two 
similar algorithms for the two attachment types. The procedures started with randomly picking a void-scaffold interface. From this starting face the algorithms tried to propagate cell material through the scaffold void in a randomly chosen direction. If an entire cell could not be propagated without hitting an obstacle in the form of scaffold material or an already seeded cell then the seeding attempt of this cell was aborted and a new propagation direction or a new starting voidscaffold interface was picked. This procedure was repeated until all cells were successfully seeded on the scaffold. The main difference between the two seeding algorithms was that for bridging cells a potential opposite attachment face was determined before the propagation attempt was started. The opposite attachment face was computed using the normal vectors of the attachment faces, the minimum and maximum cell length. In total approximately 900 (675 bridging and 225 flat) cells were numerically seeded on the used sub-volume which corresponds to the experimental setup, where $2 \times 10^{6}$ cells had been used on scaffolds with a volume of $0.443 \mathrm{~cm}^{3}$. The model of the cellseeded scaffold (sub-volume number two) is shown in Figure 4. The final geometry had a volume of $640 \mu \mathrm{m} \times 640 \mu \mathrm{m} \times 480 \mu \mathrm{m}=0.197 \mathrm{~mm}^{3}$ of which $2.2 \%, 9.6 \%$, and $88.2 \%$ were cells, scaffold material, and void, respectively.

Step 3: A smoothing procedure of the cell-seeded structure and the creation of the mesh were performed. A hexahedral mesh containing approximately 1,700,000 hexahedrons was created from the interstice of the structure. This mesh was used in the CFD simulation. Mesh refinement analyses of the CFD mesh showed that the maximum error on wall shear stress and hydrostatic pressure was below 1.5\%. Furthermore, for each cell a hexahedral mesh was calculated from the structure. The cell meshes consisted of roughly 2,200 hexahedrons and were used separately in the deformation simulation. The maximum error on displacement and von Mises stress of the elastostatics simulation was investigated by a mesh refinement analyses and was below $1 \%$.

Step 4: A CFD simulation with the following parameters and boundary conditions was performed: laminar fluid flow, incompressible Newtonian fluid with the viscosity of water at $37^{\circ}$ Celsius, noslip boundary conditions on walls, constant velocity inlet $(235 \mu \mathrm{m} / \mathrm{s}$ which corresponds to the 
experimental maximum velocity of $1 \mathrm{ml} / \mathrm{min}$ [22]), zero-pressure outlet, and impermeable cell and scaffold walls. Using an FE deformation simulation, the Young's modulus of the whole scaffold [23] was used to determine the Young's modulus of the collagen-GAG material. In agreement with [24], the simulations showed that the Young's modulus of the collagen-GAG material of our scaffold was $>10^{6} \mathrm{~Pa}$. The obtained Young's modulus of the collagen-GAG material was used in another FE study of the scaffold which revealed that the relative deformation of the scaffold caused by the fluid flow is less than $0.2 \%$. Hence, it was assumed that the scaffold material is rigid and that the walls of the scaffold do not move during the CFD simulation. Due to the small cell volume, cell deformation contributes insignificant changes to the fluid dynamics system. Therefore, the cells were also assumed to be rigid during the CFD simulation. An inlet area of a length of $100 \mu \mathrm{m}$ was added to the simulation volume to allow the nutrition fluid to distribute freely before entering the scaffold. The simulation was performed using the FV (finite volume) solver icoFoam of the open source CFD toolbox OpenFOAM [25].

Step 5: The deformation of the cells was simulated using the wall shear stress and the hydrostatic wall pressure computed in Step 4. The cells were assumed to be of isotropic, homogeneous material with a constant Young's modulus of $1 \mathrm{kPa}[26,27]$ simplifying the complex mechanical nature of the cells to a linear elastic behavior. The nodes of cell faces touching the scaffold were set to the constraint: $\mathrm{u}_{\mathrm{x}}=\mathrm{u}_{\mathrm{y}}=\mathrm{u}_{\mathrm{z}}=0$, where $\mathrm{u}_{\mathrm{x}}, \mathrm{u}_{\mathrm{y}}$, and $\mathrm{u}_{\mathrm{z}}$ are the displacements of a node in $\mathrm{x}-, \mathrm{y}-$, and $\mathrm{z}$ direction. This constraint resulted in immobile nodes at the contact area between scaffold and cells and from a simulation point of view caused the cells to stick on the scaffold. The forces caused by the wall shear stress and the hydrostatic wall pressure were applied to the nodes of faces exposed to fluid flow. The deformation simulation was done separately for each cell and performed using the FE (finite element) solver stressFemFoam of the open source CFD toolbox OpenFOAM.

The CFD simulations were performed on a 64-Bit Linux computer cluster using 8 CPUs in parallel. The CFD simulation of one sub-volume took 78 hours. A 32-Bit Linux PC was used to perform the elastostatics simulations. The FE simulation of one cell took on average 8 minutes resulting in a run 
time of $\sim 5$ days for all 900 cells of one sub-volume. 


\section{Results}

All average values and histogram distributions presented in this study have been calculated over the three simulated sub-volumes. The maximum difference in shear stress, pressure and deformation of the 3 sub-volumes was $<4 \%$. Furthermore, all results concerning cells - as forces acting on cells

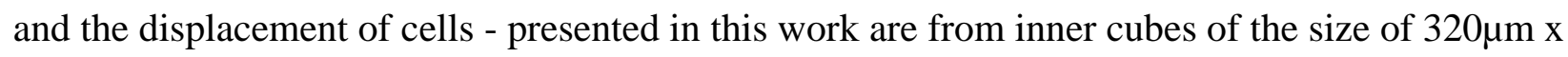
$320 \mu \mathrm{m}$ x 320 $\mu \mathrm{m}$ in order to avoid boundary artefacts [16].

Figure 5 shows (a) the magnitude of the fluid velocity and (b) the hydrostatic pressure of the culture medium inside the simulated volume (sub-volume number two). As a result of the complex scaffold geometry the fluid velocity widely varied from $0 \mu \mathrm{m} / \mathrm{s}$ to a maximum of $1425 \mu \mathrm{m} / \mathrm{s}$ with an average value of $281 \mu \mathrm{m} / \mathrm{s}$ (regarding all 3 sub-volumes). The minimum, maximum, and mean hydrostatic pressure obtained over all three sub-volumes was $-86 \mathrm{mPa}$, $877 \mathrm{mPa}$, and $379 \mathrm{mPa}$, respectively. As shown in the distribution of the magnitude of the fluid velocity inside the cell-seeded scaffold (Fig. 6), most of the scaffold volume was perfused with low fluid velocities (50\% of the volume is perfused with a fluid velocity less than $220 \mu \mathrm{m} / \mathrm{s}$ and less than $3 \%$ of the volume were subjected to a fluid velocity larger than $900 \mu \mathrm{m} / \mathrm{s}$ ). The pronounced peak at a velocity of $200-300 \mu \mathrm{m} / \mathrm{s}$ corresponds to the inlet velocity of $235 \mu \mathrm{m} / \mathrm{s}$.

In agreement with the distribution of the fluid velocity in Figure 6, the simulations revealed that most of the scaffold and cell walls were exposed to low values of shear stress (Fig. 7(a)). The wall shear stress ranged from 0 to $162 \mathrm{mPa}$ and less than $38 \%$ of the wall was exposed to a shear stress larger than 20mPa. However, the hydrostatic wall pressure acting on the scaffold and cell walls showed a plateau-like shape (Fig. 7(b)). The plateau-like pressure distribution is in agreement with Darcy's law, which predicts a linear increase of the pressure if the permeability of the scaffold is homogenous. The minimum and maximum hydrostatic wall pressure values were $-79 \mathrm{mPa}$ and $557 \mathrm{mPa}$, respectively.

Figure 8 depicts the average values of the maximum wall shear stress and the average value of the mean wall shear stress that the cells were exposed to. This indicates that cells were exposed to 
approximately the same maximum and the same mean wall shear stress regardless of attachment profile.

Figure 9 shows (a) the average of the maximum displacement and (b) the average of the mean displacement of the cells caused by the wall shear stress and the hydrostatic wall pressure. Unlike the wall shear stress and the wall pressure (Fig. 8), the displacement of the cells differs largely depending on the type of cell attachment. Cells attached to two struts experienced a deformation of up to 500 times higher than cells attached to only one strut (bridging cells $1.6 \mu \mathrm{m}$ compared to flatly attached cells $3.4 \mathrm{~nm}$ ). The respective contributions of the wall shear stress and the wall pressure were estimated by additionally performing the deformation simulations with the shear forces included and the pressure forces excluded and vice versa. When the two separately obtained displacement values are combined, a good agreement is found with the total displacement that was computed using both input types, the wall shear stress and the wall pressure. This indicates that the two contributions constructively superimpose and that the respective contributions of the wall shear stress and wall pressure can simply be calculated by neglecting the other input type. The wall shear stress and the wall pressure contributed approximately $47 \%$ and 53\% to the maximum and mean displacement of the cells attached to two struts. However for flatly attached cells there was an average contribution of $62 \%$ by wall shear stress and of $38 \%$ by wall pressure to the maximum and mean displacement, respectively.

The distribution of displacement maxima is shown in Figure 10. Figure 10(a) and Figure 10(b) depict the distribution of cells attached to two struts and of cells attached to one strut respectively. It can be seen that the maximum displacement in both groups of attachment varied widely and that only a minority of the cells experienced a displacement close to the absolute maximum of each attachment type. The pronounced variety of displacement maxima in both groups is a result of the variety of flow conditions and fluid velocities inside the scaffold. Depending on their position the cells were exposed to a wide range of acting forces and therefore showed a broad distribution of displacement maxima. 
Despite the fact that cells with both attachment profiles were on average subjected to the same mechanical load (Fig. 8) the cellular von Mises stress in bridging cells is much higher than in flatly attached cells. The averaged maximum cellular von Mises stress of bridging cells was 34mPa, whereas it was $1.3 \mathrm{mPa}$ for flatly attached cells. 


\section{Discussion}

The aim of this numerical study was to quantify the wall shear stress and hydrostatic wall pressure acting on cells seeded to collagen-GAG scaffolds and to analyse the effect of the two common attachment profiles on the cell deformation during flow perfusion experiments. The wall shear stress and the hydrostatic wall pressure were computed through the use of a CFD simulation and fed into an elastostatics model in order to calculate the deformation of the cells. Our study showed that the displacement of the cells is primarily determined by the cell attachment. Although cells of both attachment profiles were subjected to a similar flow environment, cells bridging two struts experienced a deformation up to 500 times higher than cells only attached to one strut. Recently, two types of computational studies have been reported which looked at the response of cells to fluid flow $[15,16,17]$. In the first type, 3D CFD models were used to calculate the shear stress acting on the walls of a scaffold without incorporating cells to the model. The forces acting on the cells were estimated by the results obtained from the CFD simulation and the deformation of the cells was not calculated $[15,16]$. In the second type, 3D FE models were utilized to calculate the deformation of cells without determining the exact fluid dynamical environment of the deformed cells [17]. The novel work presented in this article combines these two approaches. Firstly, the forces acting on the cells caused by the fluid flow were determined by a CFD simulation. In a second step these forces were used to calculate the deformation of the cells and the cellular von Mises stress.

The following three limitations should be considered in the interpretation of this work. Firstly, the cells were seeded to the scaffolds numerically, because it is not possible to do a $\mu$ CT scan of materials which are minimally radio absorbent such as collagen and living cells. Furthermore, $\mu \mathrm{CT}$ scans do not differentiate between scaffold material and cells. Confocal microscopy images of cellseeded scaffolds [19] have been used to optimize the cell morphology created by the numerical seeding algorithms. The most critical parameter was the diameter of the processes of bridging cells. Although varying the diameter had a strong effect on the displacement of bridging cells, it does not 
diminish the qualitative validity of our concept as the difference between bridging and flatly attached cells is still very pronounced for a wide range of diameters ( 1 to $8 \mu \mathrm{m})$. The second limitation was that for computational reasons a sequential and not a combined CFD-deformation simulation was used, meaning that the deformation of the cells was not fed back to the fluid dynamics model. Instead it was assumed that the flow conditions are only weakly altered by the deformation of the cells as the cells only occupy $2.5 \%$ of the scaffold's interstice. The third limitation was that the cells were assumed to be of linear isotropic and homogeneous material whose mechanical properties did not change with the applied mechanical load. This simplification did not mirror the complex structure of real cells and a more realistic model of the cellular components as suggested in [17] might further improve the precision of our simulations. The large range of the fluid velocity and the wall shear stress (Fig. 5 - 7) was as a result of the complex geometry of the scaffold. Although the flow conditions varied widely inside the scaffold, on average cells with both attachment profiles were exposed to approximately the same mechanical load (Fig. 8). However, the deformation of the cells strongly depended on the attachment type. Fig. 9 clearly shows that flatly attached cells were much less deformed than cells which were attached to two struts.

The variation in the cell deformation depending on the type of attachment may explain the fact that in 3D experiments, much lower fluid velocities are required to stimulate cells than in 2D experiments (without a scaffold) [28]. It might also explain why in 3D bioreactor experiments using scaffolds with pore sizes too large to allow cells to bridge two struts, instead forcing them to attach to one strut only thus simulating 2D experiments, higher fluid velocities are used [14]. In 2D experiments, a wall shear stress of $30 \mathrm{mPa}$ is not sufficient to stimulate osteoblastic cells to release the early bone formation marker prostaglandin $E_{2}$ [13], whereas in our 3D experiments shear stress values of $1 \mathrm{mPa}$ were sufficient [22].

In [14], an experiment with a calcium phosphate scaffold (with an average pore size of 350 $\mu m$ causing all cells to be flatly attached) was reported using a maximum fluid velocity of $55 \mathrm{~mm} / \mathrm{s}$ 
which is approximately 230 higher than the maximum fluid velocity of $235 \mu \mathrm{m} / \mathrm{s}$ we used in our experiments with the collagen-GAG scaffold (average pore size of 96 $\mu \mathrm{m}$ ) [22]. In both experiments the fluid velocities caused the cells to respond to the mechanical stimulus with the release of prostaglandin $\mathrm{E}_{2}$ indicating that the bone formation process was initiated. According to our simulations, the large difference in the fluid velocities is of the same order of magnitude as the difference in the displacement experienced by bridging and flatly attached cells when they are exposed to the same fluid velocity. Assuming that all cells in our scaffold were flatly attached, a fluid velocity in the order of $50 \mathrm{~mm} / \mathrm{s}$ would be required to obtain a similar displacement as bridging cells experience at fluid velocity of $235 \mu \mathrm{m} / \mathrm{s}$. This indicates that the requirement of different flow rates in 3D experiments of different scaffolds could solely be explained by the type of cell attachment.

The use of flow rates in 2D experiments commonly used in 3D experiments would result in very low bone formation activity as the shear forces are not high enough to activate bone formation on flatly attached cells. On the other hand, the use of flow rates in 3D experiments similar to values in 2D experiments would result in the loss of a significant proportion of the cells, because the high flow rates would wash many of the bridging cells off the scaffold.

Taken together, the results indicate that the cell morphology - in particular the type of cell attachment - has a major impact on the magnitude of the deformation of the cells and thereby on the activation of the bone formation process. Cells attached flatly to one strut and cells bridging two struts were exposed to approximately the same wall shear stress and the same hydrostatic wall pressure. However, the maximum displacement values of both types of cells varied significantly: single strut cells showed an average maximum displacement of $3.4 \mathrm{~nm}$, whereas cells attached to two struts experienced an average maximum displacement of $1.6 \mu m$.

The pore size of scaffolds for tissue engineering is very important. The optimal pore size must allow the cells to migrate into the scaffold and provide the cells with a sufficient amount of nutrition. Furthermore, the pore size determines the mechanical load the cells are exposed to and the way the 
cells are attached to the scaffold. As shown in the present work the sensitivity of the cells to the mechanical load applied by a fluid flow dramatically changes with the type of the attachment. Hence, the design of the optimal scaffold must take into account the interplay of the mechanical load and the type of attachment and requires a design process that optimizes both parameters at the same time. 


\section{Acknowledgements}

We are very grateful to Dr. Alex Lennon for his technical support and assistance with the Linux computer cluster.

This project was funded by a Science Foundation Ireland Industry Research Partnership with Siemens. 


\section{Conflict of Interest}

All authors declare they have no financial or personal relationships with other people or organizations that could inappropriately influence results presented here. 


\section{References}

[1] American Association of Orthopaedic Surgeons Report 2002

[2] F. R. A. J. Rose and R. O. C. Oreffo, Bone Tissue Engineering: Hope vs Hype, Biochemical and Biophysical Research Communications 292:1-7, 2002

[3] E. Wintermantel and Suk-Woo Ha, "Medizintechnik mit biokompatiblen Werkstoffen und Verfahren", Springer Verlag, ISBN: 978-3540412618, 2002

[4] A. G. Mikos and J. S. Temenoff, Formation of highly porous biodegradable scaffolds for tissue engineering, EJB Electronic Journal of Biotechnology 3(2): 1-6, 2000

[5] I. V. Yannas, E. Lee, D. P. Orgill, E. M. Skrabut, and G. F. Murphy, Synthesis and Characterization of a Model Extracellular Matrix that Induces Partial Regeneration of Adult Mammalian Skin, Proc Natl Acad Sci U S A 86:933-937, 1989

[6] F. J. O'Brien, B. A. Harley, I. V. Yannas, and L. Gibson, Influence of freezing rate on pore structure in freeze-dried collagen-GAG scaffolds, Biomaterials 25:1077-1086, 2004

[7] E. Farrell, F. J. O'Brien, P. Doyle, J. Fischer, I. V. Yannas, B. A. Harley, B. O'Connell, P. J. Prendergast, and V. A. Campbell, A Collagen-glycosaminoglycan Scaffold Supports Adult Rat Mesenchymal Stem Cell Differentiation Along Osteogenic and Chondrogenic Routes, Tissue Engineering 12(3): 459-468, 2006

[8] C. C. Compton, C. E. Butler, I. V. Yannas, G. Warland, and Dennis P. Orgill, Organized Skin Structure Is Regenerated In Vivo from Collagen-GAG Matrices Seeded with Autologous Keratinocytes, J Invest Dermatol, 110(6): 908-916, 1998

[9] G. N. Bancroft, V. I. Sikavitsas, J. van den Dolder, T. L. Sheffield, C. G. Ambrose, J. A. Jansen, and A. G. Mikos, Fluid flow increases mineralized matrix deposition in 3D perfusion culture of marrow stromal osteoblasts in a dose-dependent manner, Proc Natl Acad Sci USA 99(20):12600-5, 2002

[10] S. H. Cartmell, B. D. Porter, A. J. Garcia, and R. E. Guldberg, Effects of Media Perfusion Rate on Cell Seeded 3D Bone Constructs In Vitro, Tissue Engineering 9(6):1197-1203, 2003

[11] M. J. Jaasma, N. A. Plunkett, and F. J. O'Brien, Design and validation of a dynamic flow perfusion bioreactor for use with compliant tissue engineering scaffolds, J Biotechnology, Accepted for Publication, 2007

[12] P. J. Prendergast, R. Huiskes, and K. Søballe, Biophysical stimuli on cells during tissue differentiation at implant interfaces, J Biomech. 30(6):539-548, 1997

[13] R. Smalt F. T. Mitchell, R. L. Howard, and T. J. Chambers, Induction of $\mathrm{NO}$ and prostaglandin $\mathrm{E}_{2}$ in osteoblasts by wall-shear stress but not mechanical strain, Am J Physiol Endocrinol Metab, 273(4): E751 - 
E758: 1997

[14] J. Vance, D. Galley, D. F. Liu, and S. W. Donahue., Mechanical Stimulation of MC3T3 Osteoblastic Cells in a Bone Tissue-Engineering Bioreactor Enhances Prostaglandin $E_{2}$ Release, Tissue Engineering 11,18321839, 2005

[15] B. Porter, R. Zauel, H. Stockman, R. Guldberg, and D. Fyhrie, 3-D computational modeling of media flow through scaffolds in a perfusion bioreactor, J Biomech 38(3):543-549, 2005

[16] M. Cioffi, F. Boschetti, M. T. Raimondi, and G. Dubini, Modeling Evaluation of the Fluid-Dynamic Microenvironment in Tissue-Engineered Constructs: A Micro-CT Based Model, Biotechnology and Bioengineering, 93(3): 500-510, 2005

[17] J. G. McGarry, J. Klein-Nulend, M. G. Mullender, and P. J. Prendergast, A comparison of strain and fluid shear stress in stimulationg bone cell responses - a computational and experimental study, FASEB Journal 19(3):482-4, 2005

[18] T. Gupta and T. L. H. Donahue, Role of cell location and morphology in the mechanical environment around meniscal cells, Acta Biomaterialia 2(5): 483-492, 2006

[19] L. McMahon, The effect of cyclic tensile loading and growth factors on the chondrogenic differentiation of bone-marrow derived mesenchymal stem cells in a collagen-glycosaminoglycan scaffold, PhD thesis, Trinity College Dublin, 2007

[20] F. J. O'Brien, B. A. Harley, I. V. Yannas, and L. Gibson, The effect of pore size on cell adhesion in collagen-GAG scaffolds, Biomaterials 26(4): 433-441, 2005

[21] M. Manimaran, F. E. H. Tay, and K. C. Chaw, Cell Deformation in Cancer Metastasis: a BioMEMS Based Approach, Journal of Physics: Conference Series 34: 1143-1147, 2006

[22] M. J. Jaasma and F. J. O'Brien, Mechanical stimulation of osteoblasts by steady and dynamic fluid flow, Tissue Engineering, In Review, 2007

[23] M. G. Haugh, M. J. Jaasma, and F. J. O'Brien, The Effect of Dehydrothermal Treatment on the Mechanical and Structural Properties of Collagen-GAG scaffolds, Journal of Biomedical Materials Research Part A, accepted, 2007

[24] B. A. Harley, J. H. Leung, E. C. C. M. Silva, and L. J. Gibson, Mechanical characterization of collagenglycosaminoglycan scaffolds, Acta biomaterialia 3(4), 463-474, 2007

[25] http://WWW.opencfd.co.uk/openfoam/

[26] H. Karcher, J. Lammerding, H. Huang, R. T. Lee, R. D. Kamm, and M. R. Kaazempur-Mofrad, A ThreeDimensional Viscoelastic Model for Cell Deformation with Experimental Verification, Biophysical Journal, 85, 
3336-3349, 2003

[27] D. P. Theret, M. J. Levesque, F. Sato, R. M. Nerem, and L. T. Wheeler, The application of a homogenous half-space model in the analysis of endothelial cell micropipette measurements, $\mathrm{J}$ Biomech Eng. 110(3): 190-199, 1998

[28] N. Batra, Y. Li, C. Yellowley, L. You, A. Malone, C. Kim, and C. Jacobs, Effects of short-term recovery periods on fluid-induced signaling in osteoblastic cells, J Biomech 38:1909-1917, 2005

[29] L.E. Claes and C.A. Heigele, Magnitudes of local stress and strain along bony surfaces predict the course and type of fracture healing, J Biomechanics 32: 255—266, 1999

[30] D.R. Carter, G.S. Beaupré, N.J. Giori and J.A. Helms, Mechanobiology of skeletal regeneration, Clinical Orthopaedics and Related Research 355S: S41-S55, 1988

[31] Y. Wang, L.M. McNamara, M. B. Schaffler, and S. Weinbaum, A model for the role of integrins in flow induced mechanotranduction in osteocytes. PNAS 104(40), 15941, 2007

[32] S. Weinbaum, S. C. Cowin, and Y. Zeng, A model for the excitation of osteocytes by mechanical loading-induced bone fluid shear stresses. J. Biomech. 27, 339, 1994

[33] U. Meyer, B. Kruse-Lösler, and H.P. Wiesmann, Principles of bone formation driven by biophysical forces in craniofacial surgery. British Journal of Oral and Maxillofacial Surgery 44: 289-295, 2006

[34] C. Sandino, J. A. Planell, and D. Lacroix, A finite element study of mechanical stimuli in scaffolds for bone tissue engineering 41: 1005-1014, 2008

[35] T. M. Freyman, I. V. Yannas, Y-S. Pek, R. Yokoo, and L. J. Gibson, Micromechanics of Fibroblast Contraction of a Collagen-GAG Matrix. Experimental Cell Research 269: 140, 2001 
Fig. 1: Flow perfusion bioreactor used in our laboratory to apply biophysical stimuli and thereby increase the mechanical properties of cell-seeded collagen-GAG scaffolds [11,22].

Fig. 2: $\mu \mathrm{CT}$ scan of the unseeded collagen-GAG scaffold used to reconstruct the 3D geometry for the sequential CFD-deformation simulation.

Fig. 3: The typical geometry of (a) a numerically seeded cell attached to two struts and of (b) a cell flatly attached to one strut - blue elements are scaffold material, red elements are cells.

Fig. 4: 3D mesh of the reconstructed cell-seeded collagen-GAG scaffold (pore size $~ 96 \mu \mathrm{m}$ ) used to perform the CFD simulation (sub-volume number two) - blue elements are scaffold material, red elements are cells.

Fig. 5: The magnitude of the fluid velocity (a) and the hydrostatic pressure (b) of the culture medium inside the simulation volume (sub-volume number two) in $\mathrm{x}-\mathrm{z}$ plane at $\mathrm{y}=320 \mu \mathrm{m}$ for an inlet velocity of $235 \mu \mathrm{m} / \mathrm{s}$.

Fig. 6: Histogram distribution of the velocity of the culture medium inside the scaffold for an inlet velocity of $235 \mu \mathrm{m} / \mathrm{s}$ taking into account all three simulated sub-volumes (without inlet area).

Fig. 7: Histogram distribution of (a) the wall shear stress and (b) the hydrostatic wall pressure of all three simulated sub-volumes acting on the walls (scaffold and cells).

Fig. 8: The average of the maximum and mean wall shear stress acting on the cells. The diagram was created in a two step process. First, the maximum wall shear stress and the mean wall shear stress were determined for each cell. Then the maximum wall shear stress (max) and the mean wall 
shear stress (min) were averaged over all cells of an attachment group (taking into account only cells inside the three $320 \mu \mathrm{m}$ x 320 $\mu \mathrm{m}$ x 320 $\mu \mathrm{m}$ inner cubes).

Fig. 9: The average displacement of cells attached to two struts (a) and to one strut (b). (max) and (mean) display the average of the maximum and mean displacement of the cells, respectively. The average was taken over all cells of a certain attachment type inside the three $320 \mu \mathrm{m}$ x $320 \mu \mathrm{m}$ x $320 \mu \mathrm{m}$ inner cubes. Note that the displacement in (a) is shown in [ $\mu \mathrm{m}]$ and in (b) in [nm].

Fig. 10: Histogram distribution of the maximum displacement of cells attached to two struts (a) and cells flatly attached to one strut (b). Note that the maximum displacement in (a) is shown in $\mu \mathrm{m}$ and in (b) is shown in $\mathrm{nm}$. 
Figure 1

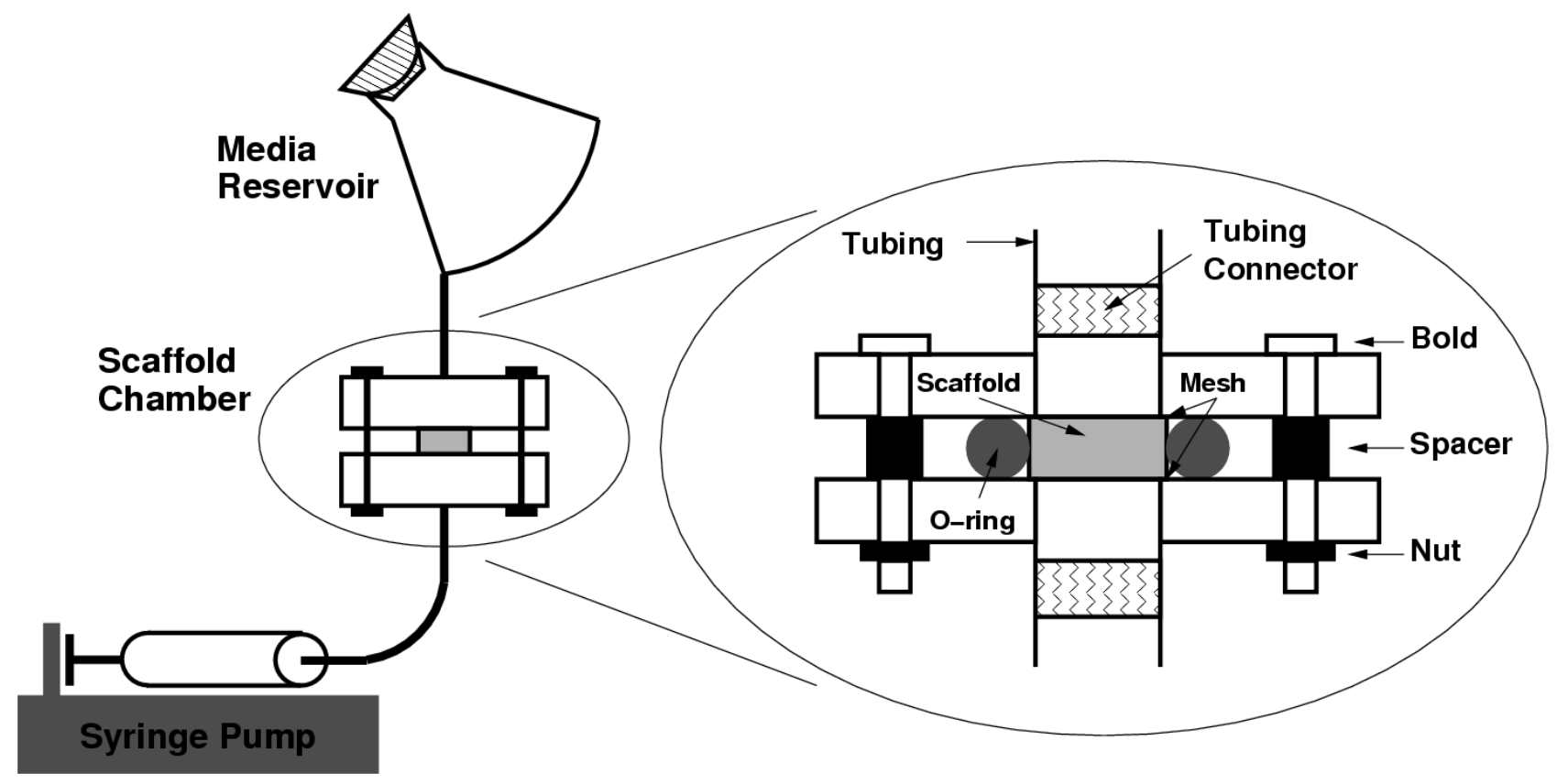




\section{Figure 2}

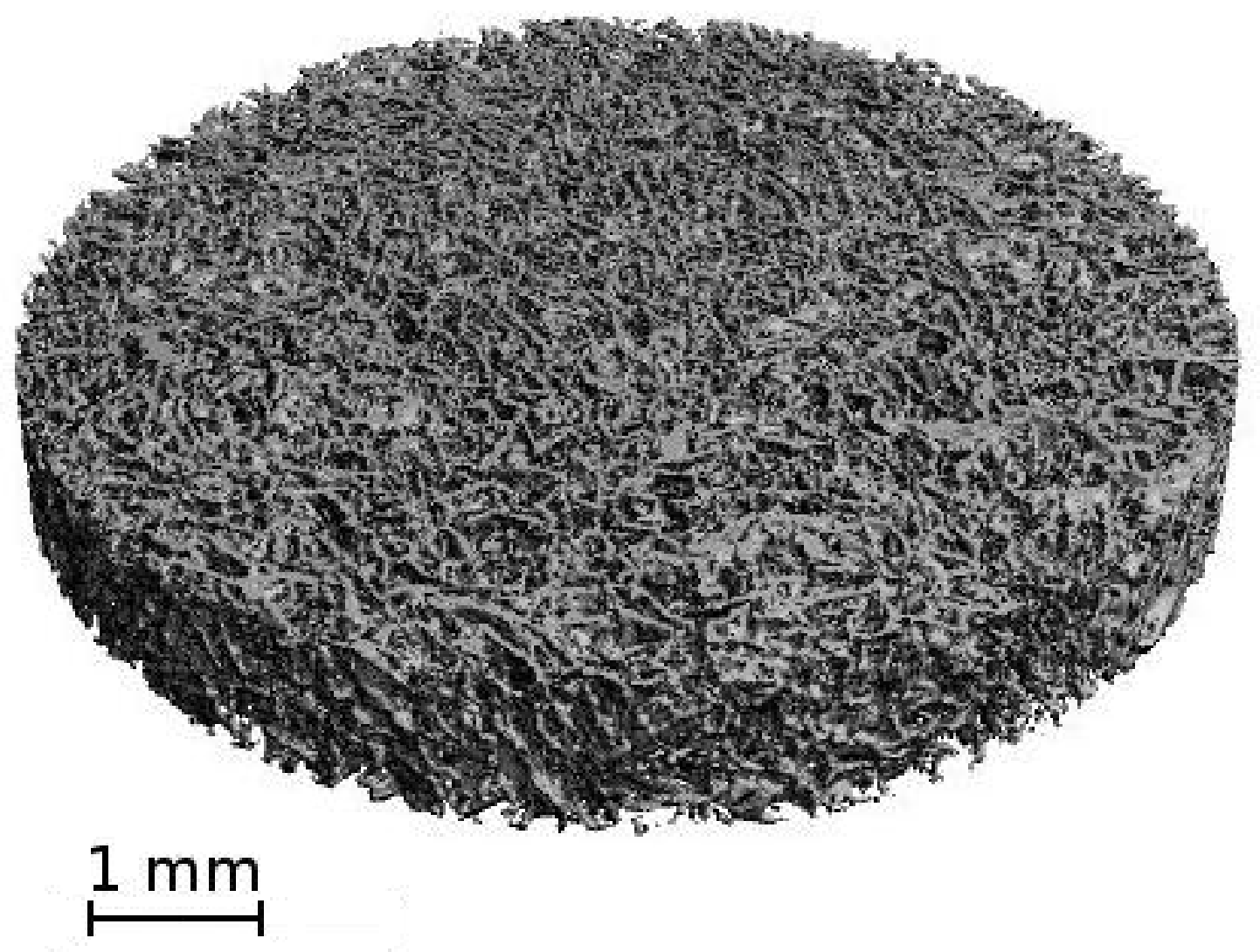


Figure 3
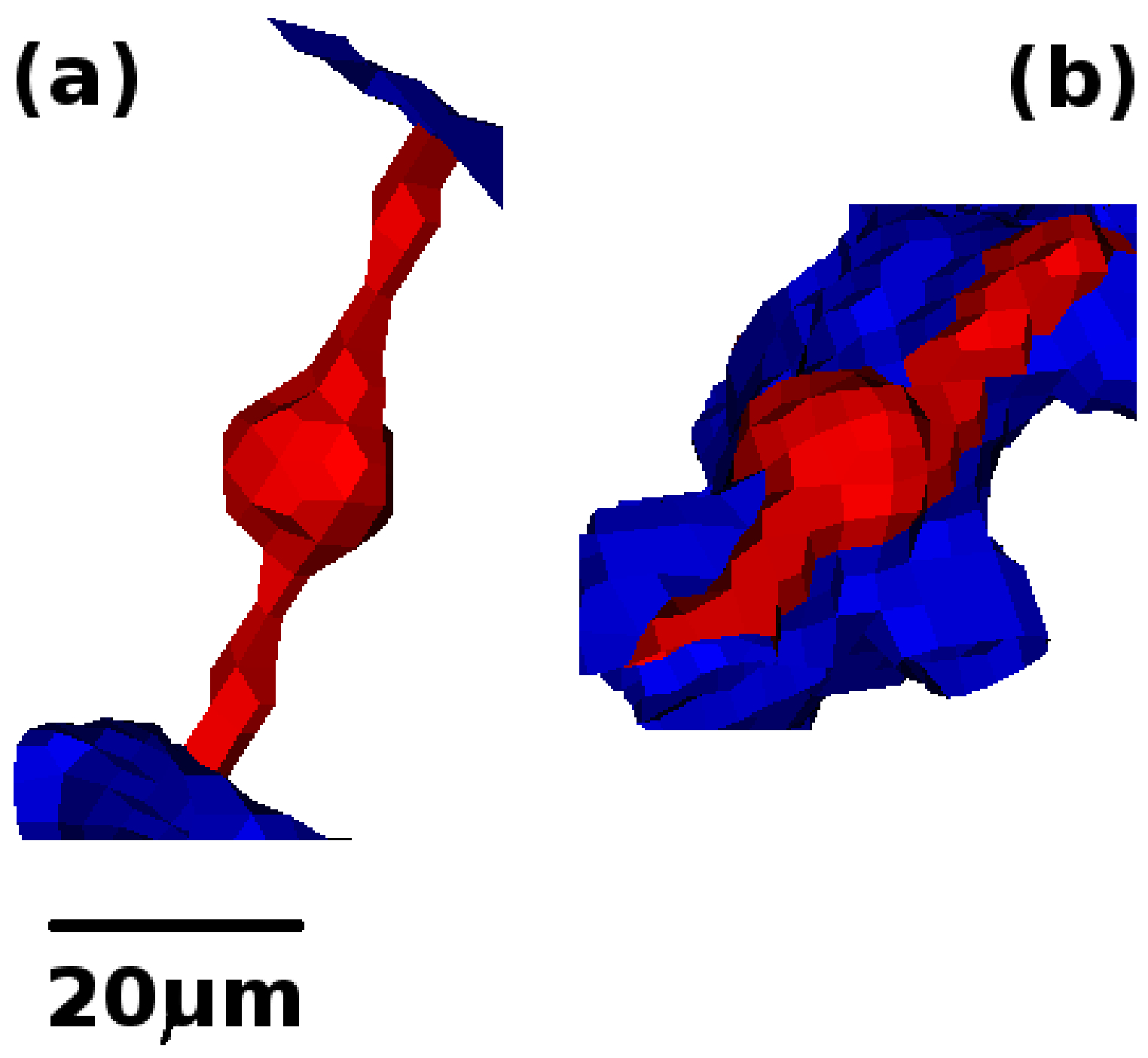

$20 \mu \mathrm{m}$ 
Figure 4

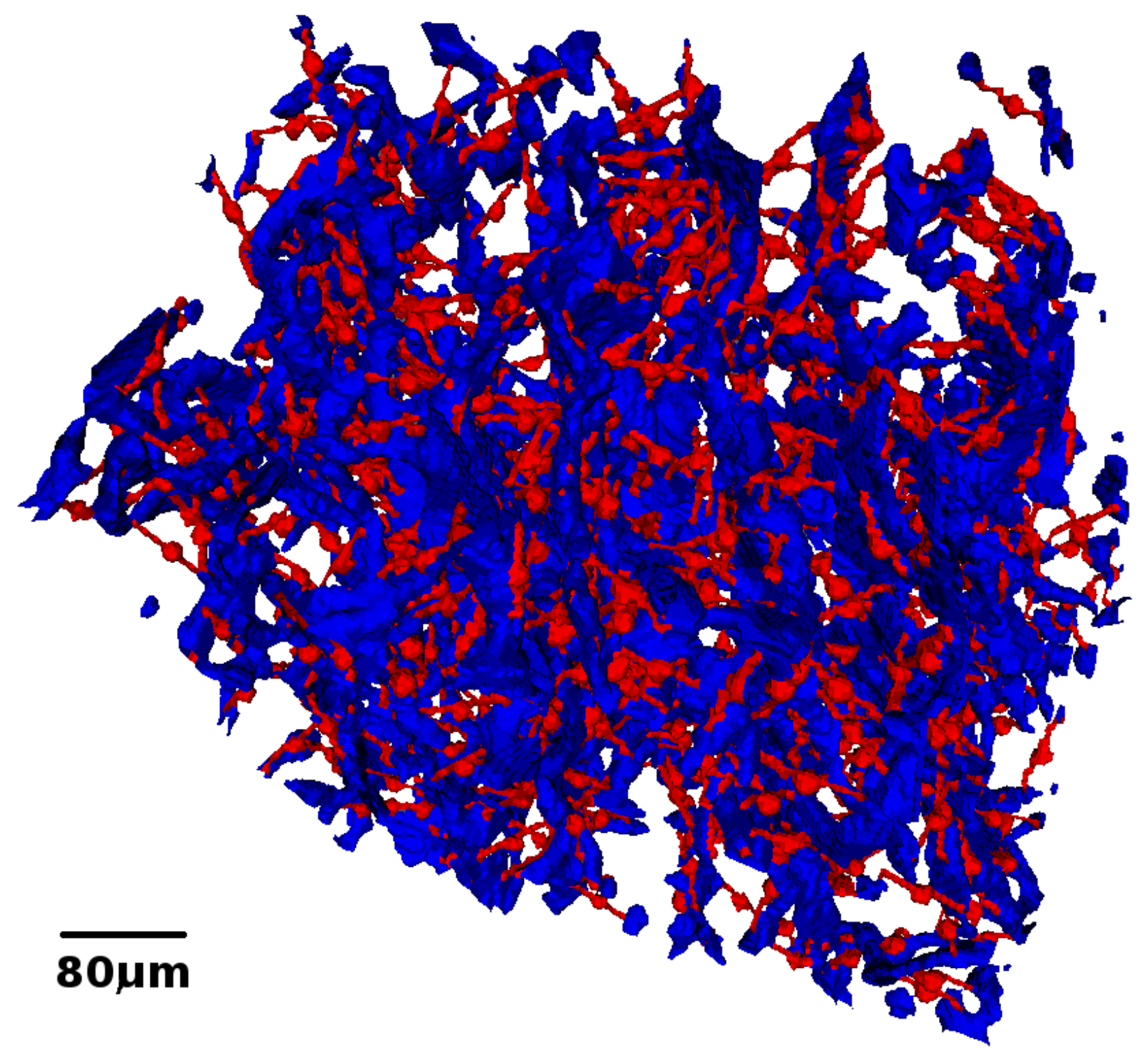


Figure 5

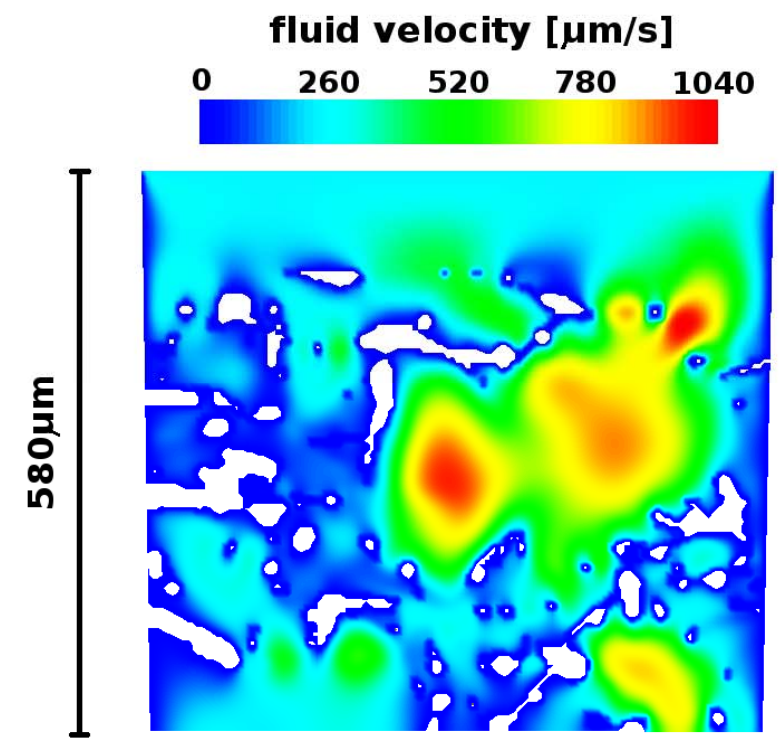

(a) hydrostatic pressure [mPa]

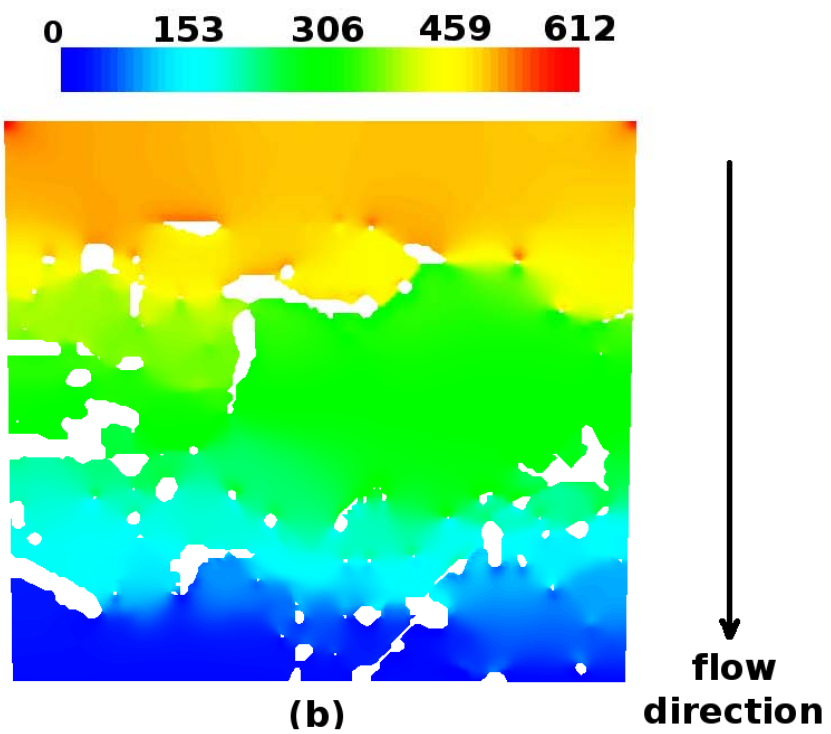


Figure 6

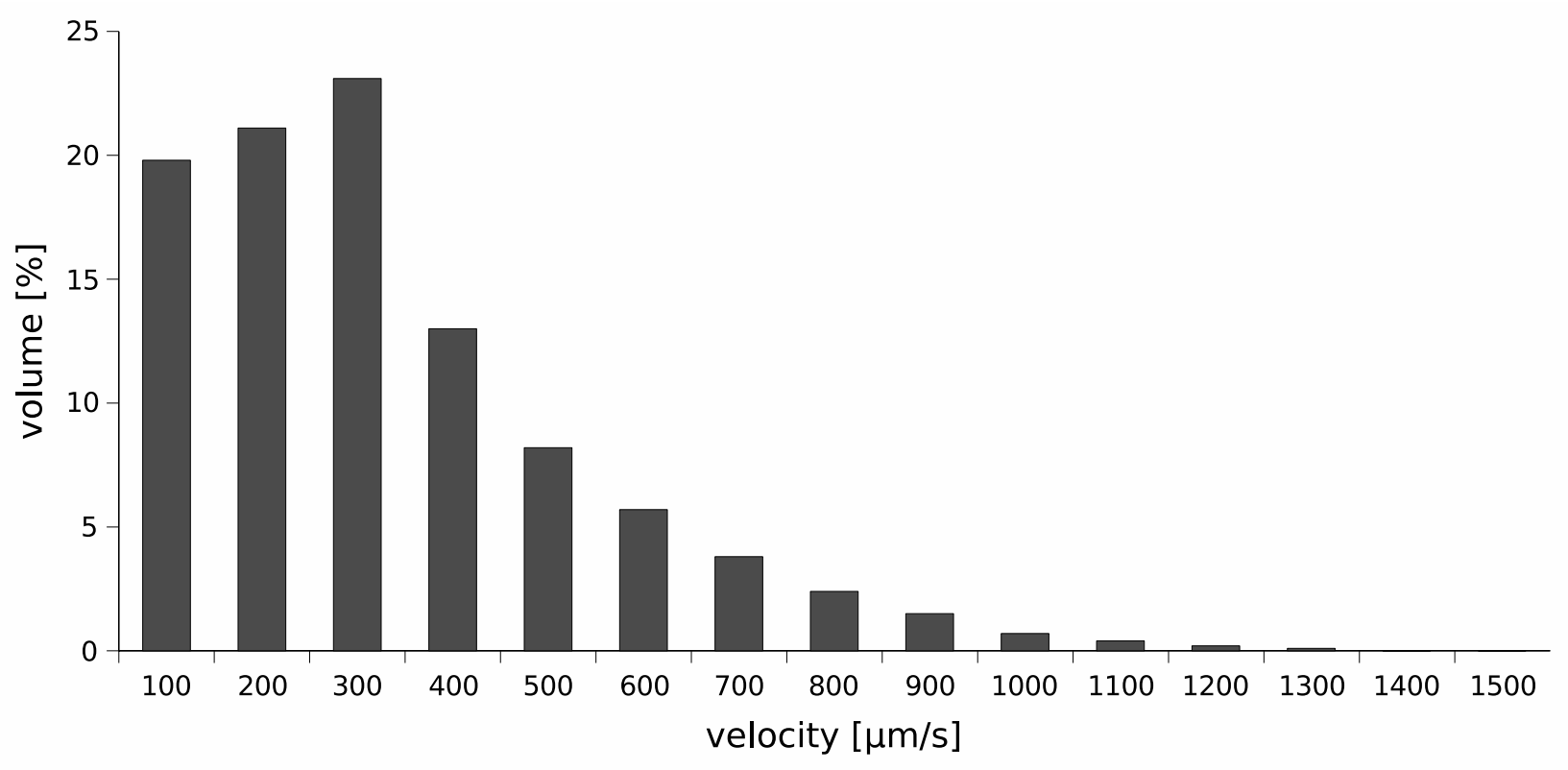


Figure 7
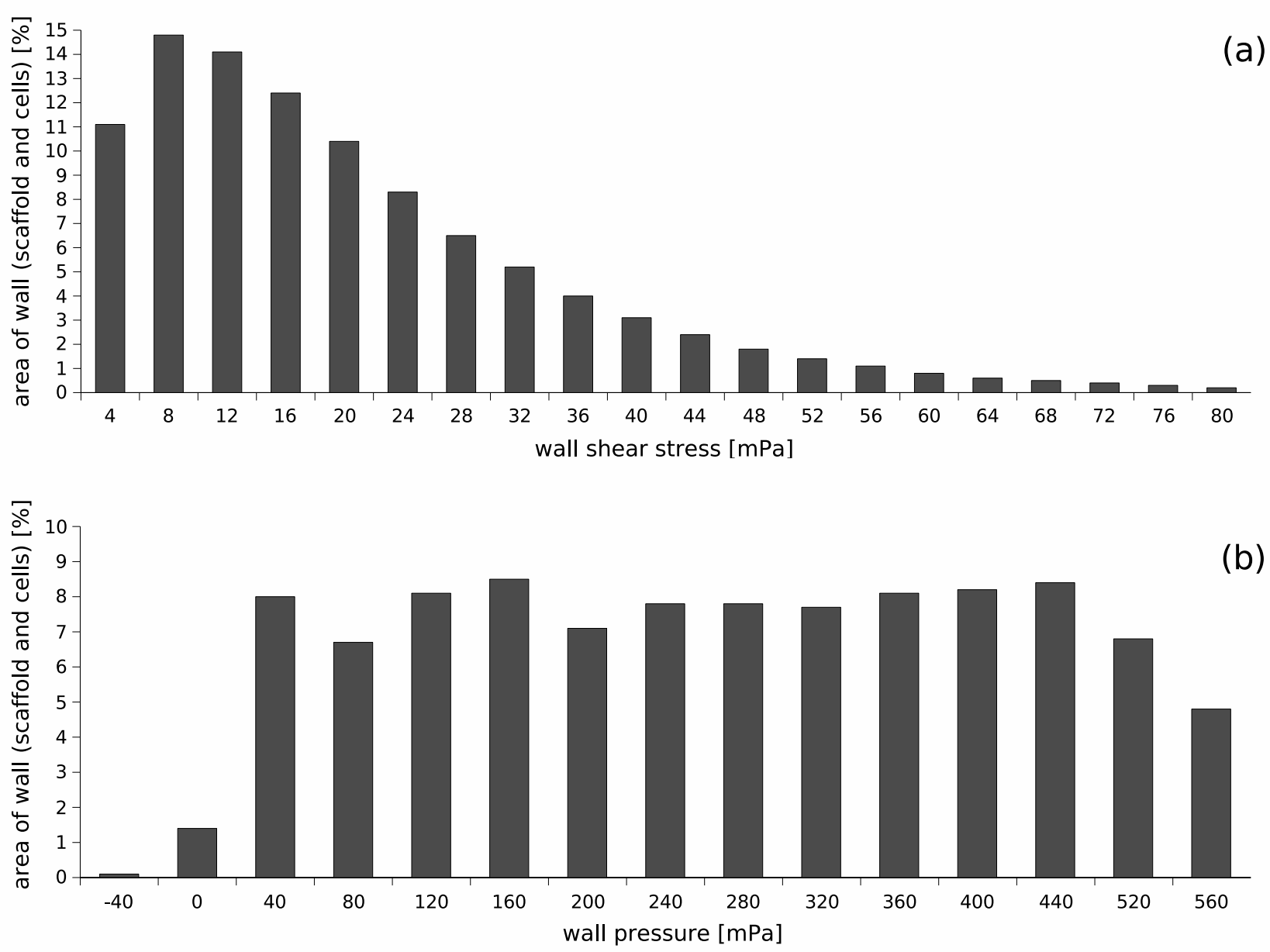
Figure 8

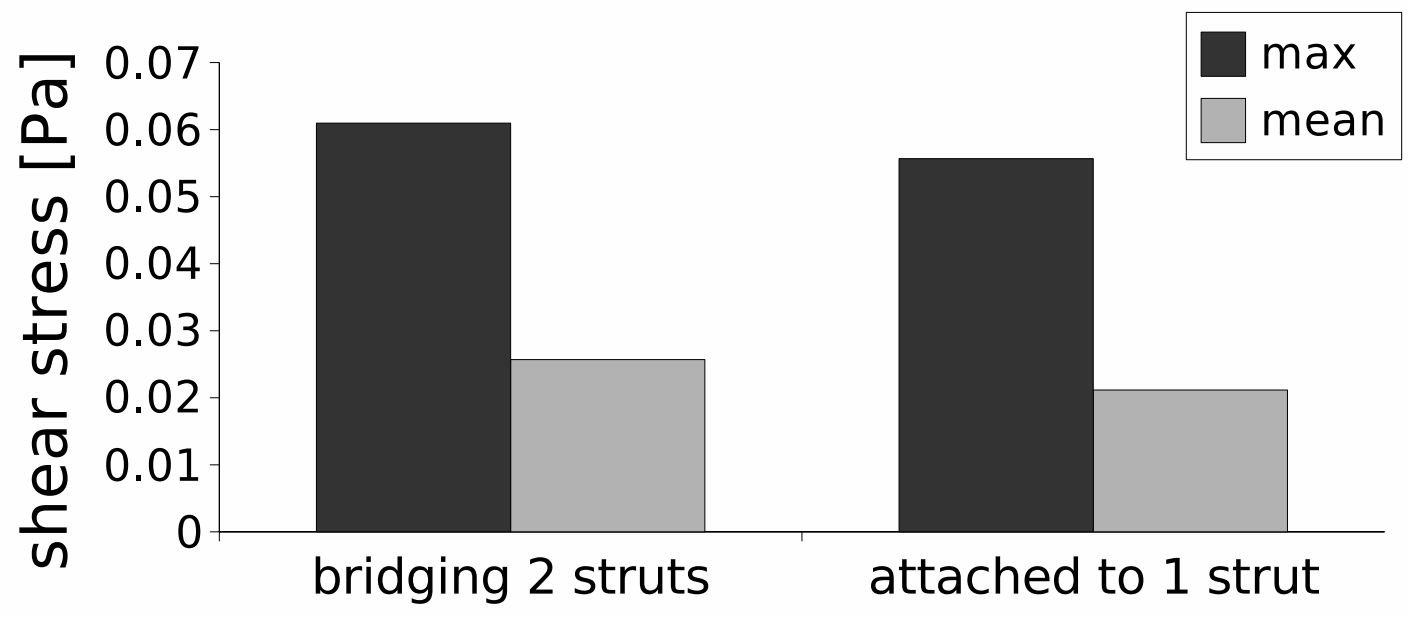


Figure 9
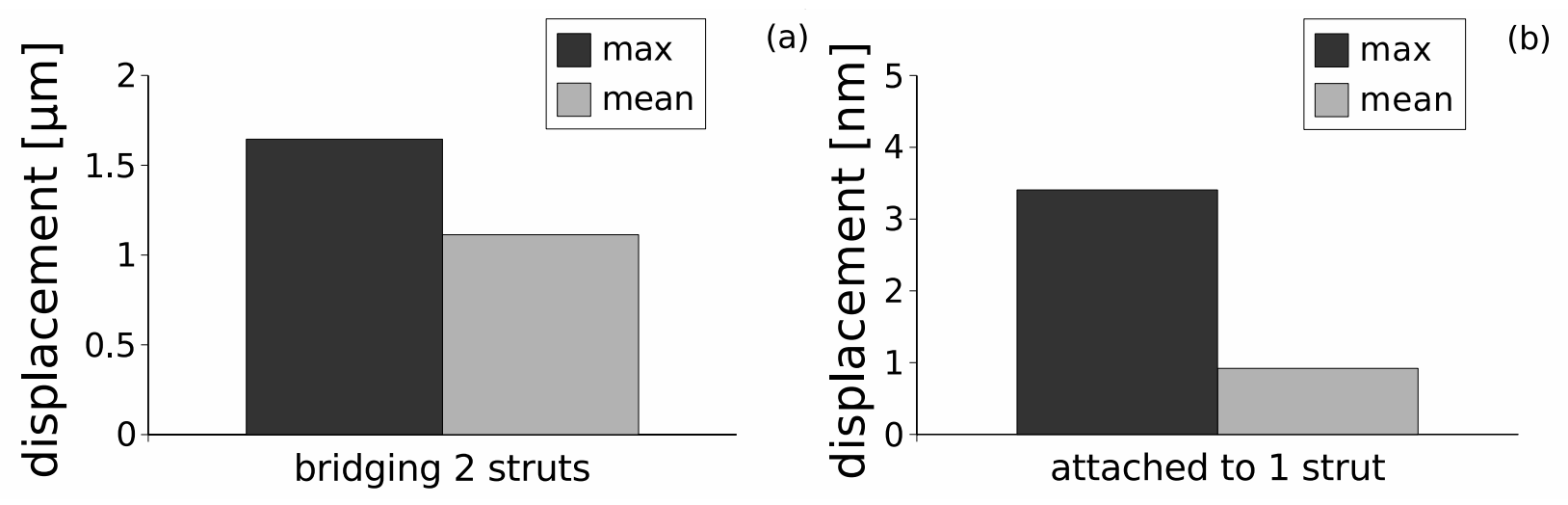
Figure 10
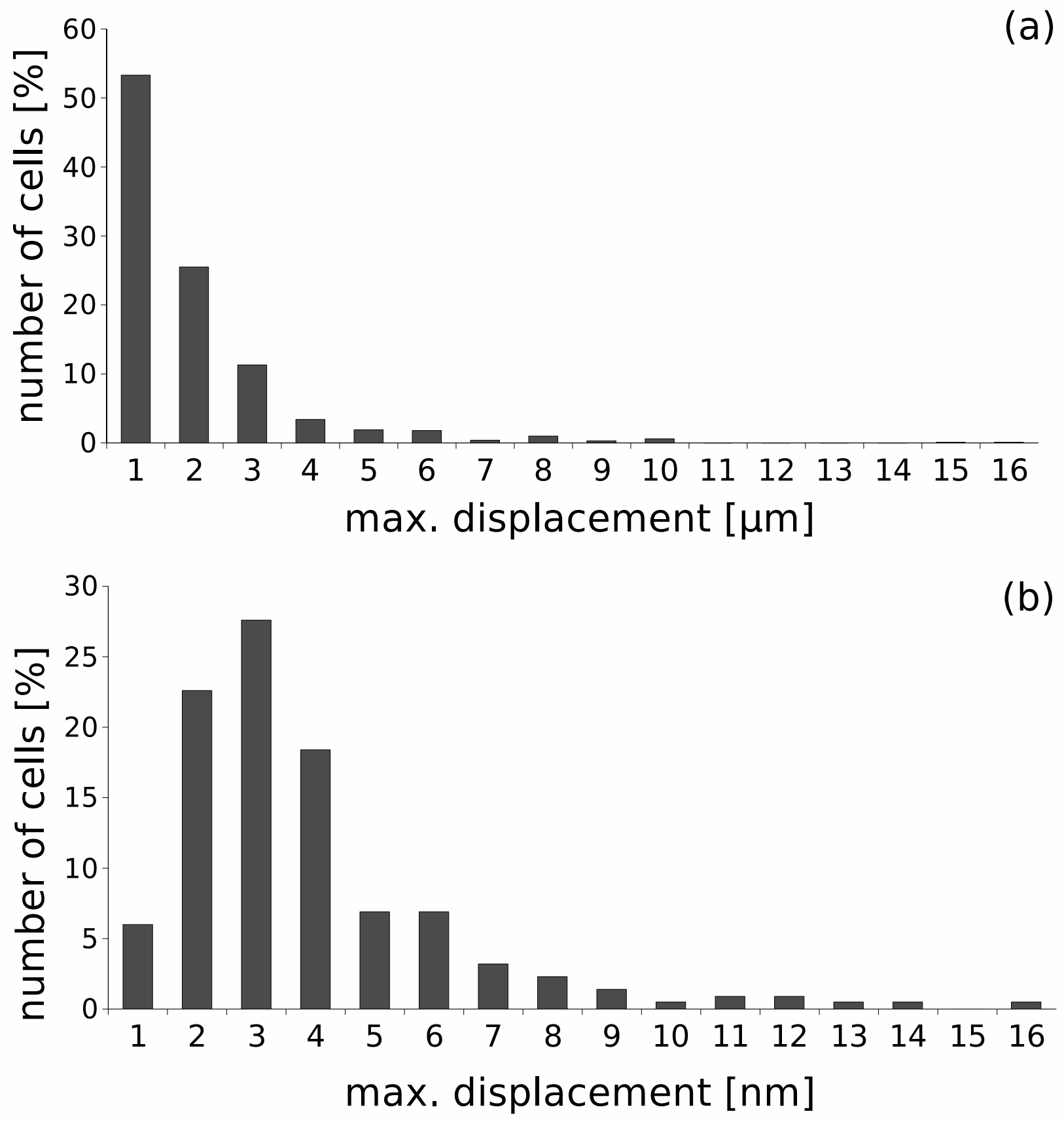